\title{
Intraoperative Neurophysiologic Sensorimotor Mapping-A Review
}

\section{Mirela V. Simon*}

Department of Neurology, Massachusetts General Hospital, Harvard Medical School, 55 Fruit St, Boston

\section{Introduction}

The main goal of neurological surgery for supratentorial lesions remains maximal resection with preservation of function of the nearby eloquent cortical and subcortical structures. Many authors [1-9] have emphasized the positive impact of aggresive removal of gliomas on the survival rate and the quality of life in both adults and children. Others [10-12] have found a positive correlation between incomplete resection of an epileptic focus and poor seizure control outcome in epilepsy surgery.

However, maximal resection of supratentorial lesions is in many circumstances difficult to achieve due to the close proximity of functionally normal eloquent cortex. Despite advanced neuroimaging techniques and sensitive microscopes, visual inspection has often suboptimal resolution for distinguishing between normal and abnormal tissue, especially in cases of distorted anatomy and infiltrative pathology. More so, abnormal brain tissue, as appreciated by visual inspection, can retain normal function [13-15]. Thus, functional cortical mapping is essential for safe resection of lesions in the vicinity of eloquent cortex. This usually entails a multimodality approach, including functional magnetic resonance imaging (fMRI), positron emission tomography (PET), diffusion tensor imaging (DTI) and neurophysiologic studies as well as co-registration techniques that optimally utilize all the available data $[16,17]$.

This article offers an overview of the neurophysiologic sensorimotor mapping. Probably one of the most important advantage this method has over the neuroimaging techniques is allowing live assessment of the cortical function and direct intraoperative feedback to the surgeon. Unlike fMRI, neurophysiologic techniques also allow subcortical mapping [18] and continuous monitoring of the sensorimotor pathways during the actual resection; also, its results are much less affected by the perilesional hemodynamic changes. Last, neurophysiologic mapping offers increased localizing specificity, when compared to other techniques $[19,20]$.

Sensorimotor neurophysiologic mapping consists of two parts: first, contralateral (to the craniotomy side) median somatosensory evoked potentials (SSEPs) phase reversal technique is employed with the attempt to localize the central sulcus (CS). Identification of the latter reveals to the surgeon and neurophysiologist the presumed location of the primary motor cortex. In the second part of the motor mapping, the surgeon stimulates the precentral regions, in order to identify the motor strip. Direct electrical stimulation is applied subdurally or epidurally and triggered muscle motor evoked responses (mMEPs) and/or evoked clinical responses are recorded and/or observed in the contralateral hemibody muscles in anesthetized or awake patients. Triggered responses at the lowest current amplitude will help delineate the primary motor cortex. Additionally, once the motor strip is identified, its continuous stimulation allows monitoring of the primary cortex and corticospinal tract throughout the resection. Similarly, recording of the thalamocortical SSEPs helps identification of the somatosensory cortex and monitoring of the large fiber sensory pathways during lesionectomy. Last, in awake patients, somatosensory cortex can be further delineated by eliciting sensory symptoms by electrical stimulation of the postcentral regions.

\section{Central Sulcus Localization}

\section{Principle}

At our institution, sensorimotor mapping starts with accurate identification of the CS. This can be sometimes achieved by appreciation of the anatomic landmarks on MRI brain images [21] However, distorted anatomy as well as neurophysiologic-neuroanatomic dissociation can occur in cases of infiltrative and/or large pericentral lesions. Thus, one of the most reliable ways to identify the CS is via median SSEPs phase reversal technique $[16,22,23]$.

It is known that at approximately $19 \mathrm{msec}$ from the electrical stimulation of the median nerve at the wrist, the contralateral postcentral parietal somatosensory cortex (labeled $\mathrm{Cpc}$ and corresponding to $\mathrm{Cp} 3$ or Cp4 electrode position on 10-20 International Electrode System) becomes more electronegative that its contrapart (Cpi), than the frontal regions (Fpz, Fc depicting either F3 or F4) and than the ipsilateral (to the stimulation) mastoid (Ai). Thus, in a Cpc-Cpi, $\mathrm{Cpc}-\mathrm{Fc}$ or $\mathrm{Cpc}-\mathrm{Ai}$ channel thalamocortical median SSEPs will be recorded as a negative peak with an absolute latency of approximately $19 \mathrm{msec}$ (N19). Of course, this latency varies, according to subject specific factors, such as height, presence or absence of sensory symptoms and type and depth of anesthesia. However, at the same latency as the negative parietal N19, the frontal precentral regions become more electropositive than the ipsilateral (to the stimulation site) mastoid. As a consequence, in a Fc-Ai channel, a positive peak will be recorded. Thus, simultanoues recordings of median SSEPs from the contralateral precentral and postcentral cortical regions, referenced to the ipsilateral mastoid, will reflect a phase reversal in the dipole polarity, at the level of the CS and so identification of the latter.

\section{Technical details}

Stimulation: The most commonly stimulated nerve is the median nerve, though other nerves have been also employed [22]. Repetitive pulses at $3.17 \mathrm{~Hz}, 0.3 \mathrm{msec}$ and intensity varying between $10-20 \mathrm{~mA}$ (the lowest intensity resulting in a robust thumb twitch) are used to stimulate peripherally the median nerve, usually at the wrist, via two

*Corresponding author: Mirela V Simon, WACC 739 G, Department of Neurology, Massachusetts General Hospital, Harvard Medical School, 55 Fruit St, Boston, MA 02114, Tel: 617 724-2655; Fax: 617 724-6513; E-mail: mvsimon@partners.org

Received August 08, 2011; Accepted December 02, 2011; Published December 20, 2011

Citation: Simon MV (2011) Intraoperative Neurophysiologic Sensorimotor Mapping-A Review. J Neurol Neurophysiol S3. doi:10.4172/2155-9562.S3-002

Copyright: (c) 2011 Simon MV. This is an open-access article distributed under the terms of the Creative Commons Attribution License, which permits unrestricted use, distribution, and reproduction in any medium, provided the original author and source are credited. 
stick-on electrodes (Rochester, Electro-Medical Inc). Since electrical stimulation could cause unpleasant sensations, in awake craniotomies, we always inform the patients what to expect during the stimulation.

We usually average at least several hundred trials. This is important for maximizing the signal-to-noise ratio, as direct cortical recordings are notorious for pulsation, movement and electrical artifact. Also, averaging becomes important in cases of sensory symptoms, when small amplitude, abnormal SSEPs are expected.

Recording: Thalamocortical SSEPs are recorded via an 8 contact subdural strip electrode (Ad Tech Manufacturer) placed by the surgeon either subdurally or epidurally, perpendicular to and crossing the presumed direction of the CS, on the contralateral (to the stimulated nerve) hemisphere (Figure 1). Given that we stimulate the median nerve, the desired "latitude" for the strip placement is at the level of the hand area on the hemispheric convexity. Subdermal needle (anesthetized patients) or a surface stick on (awake patients) electrodes are used as reference electrodes, placed on the ipsilateral (to the stimulated nerve) mastoid (Ai). Both bipolar as well as reference montage recordings are used. In a reference montage, the phase reversal points towards the location of the CS, whereas in a bipolar montage, the phase reversal points towards the most electronegative and most electropositive regions, relative to the adjacent contact positions, thus towards the postcentral and precentral region respectively (Figure 1).

\section{Troubleshooting}

Increased noise: Intraoperative direct cortical recordings are susceptible to both electrical (i.e., $60 \mathrm{~Hz}$ ) as well as pulsation artifact. An increase in averaged trials is recommended in such situations. In regards to the $60 \mathrm{~Hz}$ noise, one should ensure that the stimulation rate is not a number divisible by 2 or 3 . In case of significant amount of pulsation artifact, reposition of the strip, ideally away from cortical vessels, may help. Also, virtually in every mapping done at our institution, electrode artifact was present in one form or another. Irrigating the recording strip with warm saline, repositioning or replacing it, securing its position in the field, or simply disabling the defective contacts can help.

No recorded SSEPs: Absence of recorded SSEPs could mean a technical problem, use of an inappropriate anesthetic regimen (see section VII), recording away from the somatosensory cortex, or no recordable evoked responses in symptomatic patients. First, technical troubleshooting should rule out inadequate stimulation, erroneous connections or recording parameters. Second, the neurophysiologist should ensure that an appropriate anesthetic regimen is used. Once technical and anesthetic reasons are ruled out, repositioning of the recording strip should be attempted. Misplacement of the strip, away from the somatosensory cortex, is frequently encountered in cases of distorted local anatomy, when there is en bloc displacement of the pericentral regions or neuroanatomic-neurophysiologic dissociation.

\section{Interpretation}

It is our experience that perfect phase reversal with the first strip position is rarely obtained. Leaving aside the reasons specified in the troubleshooting section, this is due to the fact that an ideal position of the strip, across and perpendicular to the CS, also at the right "latitude" on the hemispheric convexity is hard to achieve on first attempt, especially when there is underlying pericentral pathology. The reader will find below some scenarios that are likely to occur in early stages of this technique.
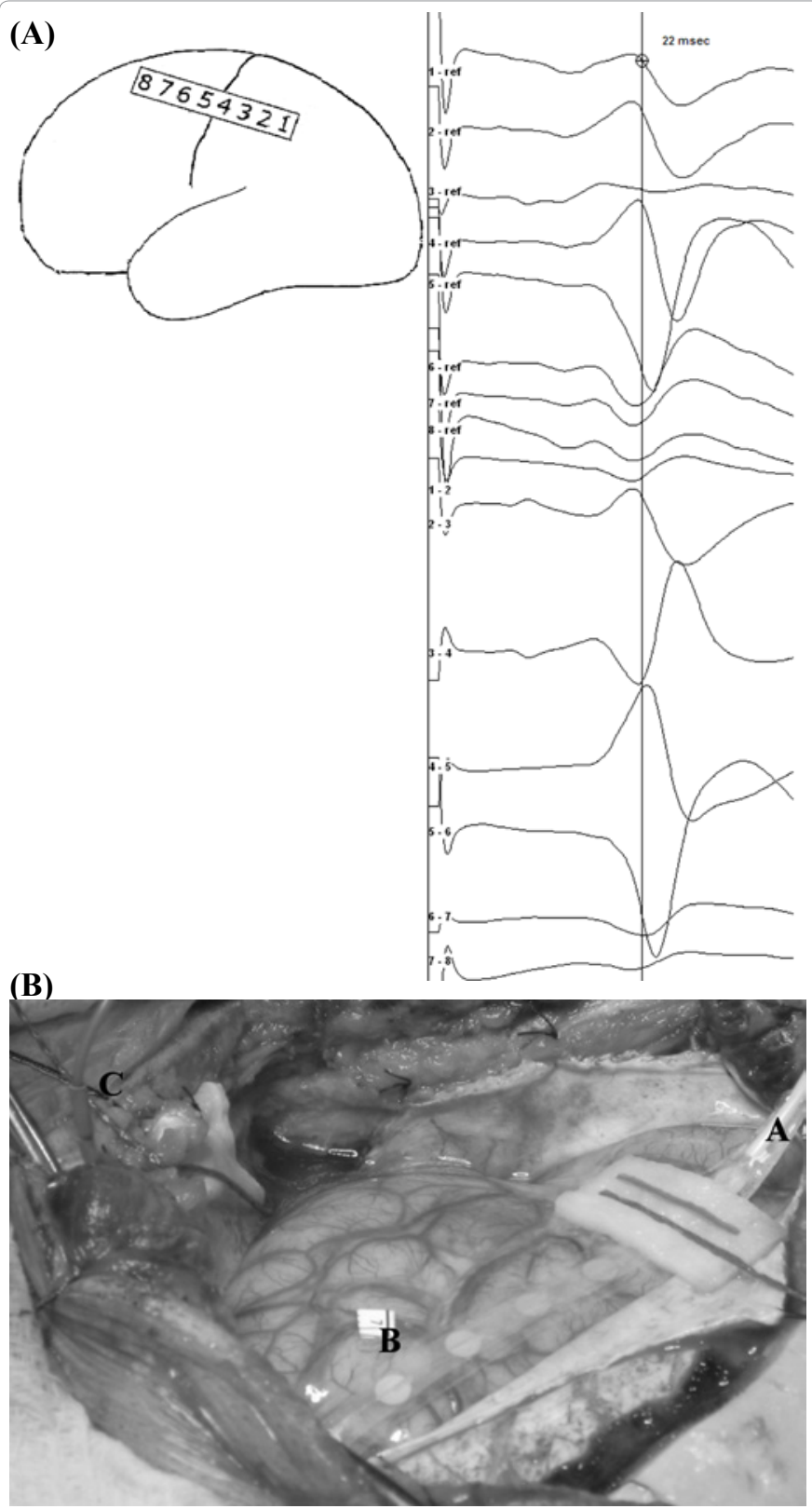

Figure 1: Recording subdural strip electrode. a- Right median SSEPS phase reversal technique. Referential and bipolar recordings, with the recording strip placed with contact 1 most posterior in the anterior-posterior axis. The referential montage shows a phase reversal at the level of CS: contacts 4 is located postcentrally and contact 5 precentrally. In the bipolar montage, notice the phase reversal at contact 4 , pointing towards the most electronegative region, the parietal somatosensory cortex.

b- Subdural 8 contact strip electrode placed directly on the cortical surface. Label A shows the extension cable, with contact 8 being the closest. Label B shows a "flag" on the cortical surface, marking eloquent cortex. Label $\mathbf{C}$ shows the sterile subdermal needle electrode placed at the margin of the surgical field and connected to the cathode.

If a negative peak is seen in all the channels of the referential montage, this should suggest that the entire strip is positioned postcentrally and hasn't crossed the CS as yet. Thus, the surgeon should be advised to push the strip forward on the anterior-posterior axis. Similarly, if a positive peak is seen instead in all channels, the recording contacts are most likely located anterior to the CS and thus the strip should be pushed posteriorly. 
Citation: Simon MV (2011) Intraoperative Neurophysiologic Sensorimotor Mapping-A Review. J Neurol Neurophysiol S3. doi:10.4172/2155-9562. S3-002

If there is a hint of change in polarity or change in morphology the strip should be 'rotated", repositioned at a different angle. Example of strip repositioning can be found in Figure 2.

\section{III.Primary Motor Cortex Localization}

\section{Principle}

Both SSEPs phase reversal technique and direct cortical electrical stimulation can be used for motor mapping [24]. In fact, we strongly recommend the use of both techniques, starting with CS localization as described in section II. This will allow a good starting point for the electrical stimulation and will increase the precision and the overall efficiency of the mapping.

Once the CS is identified, direct cortical electrical stimulation of the precentral regions with recording of the resulting motor responses, can be performed. . This technique is based on early findings of [25] who were able to record electrical volleys in the cortico-spinal tract (i.e., D waves) at the level of pyramids and medulla. These neurogenic responses can be also recorded at the level of spinal cord. Stimulation of the motor cortex also results in more distal muscle motor evoked responses (mMEPs) as well as in motor twitches/dystonia/tonic-clonic movements in the contralateral hemibody muscles. At our institution, we prefer recording mMEPs from the corresponding muscles (rather than relying on observing clinical movements), as the stimulation threshold for obtaining mMEPs is smaller than that for obtaining clinical movements; thus, the risk of stimulation triggered seizures is decreased. Also, electrophysiologic recording allows a broad sampling and simoultaneous view of the activity in many contralateral hemibody

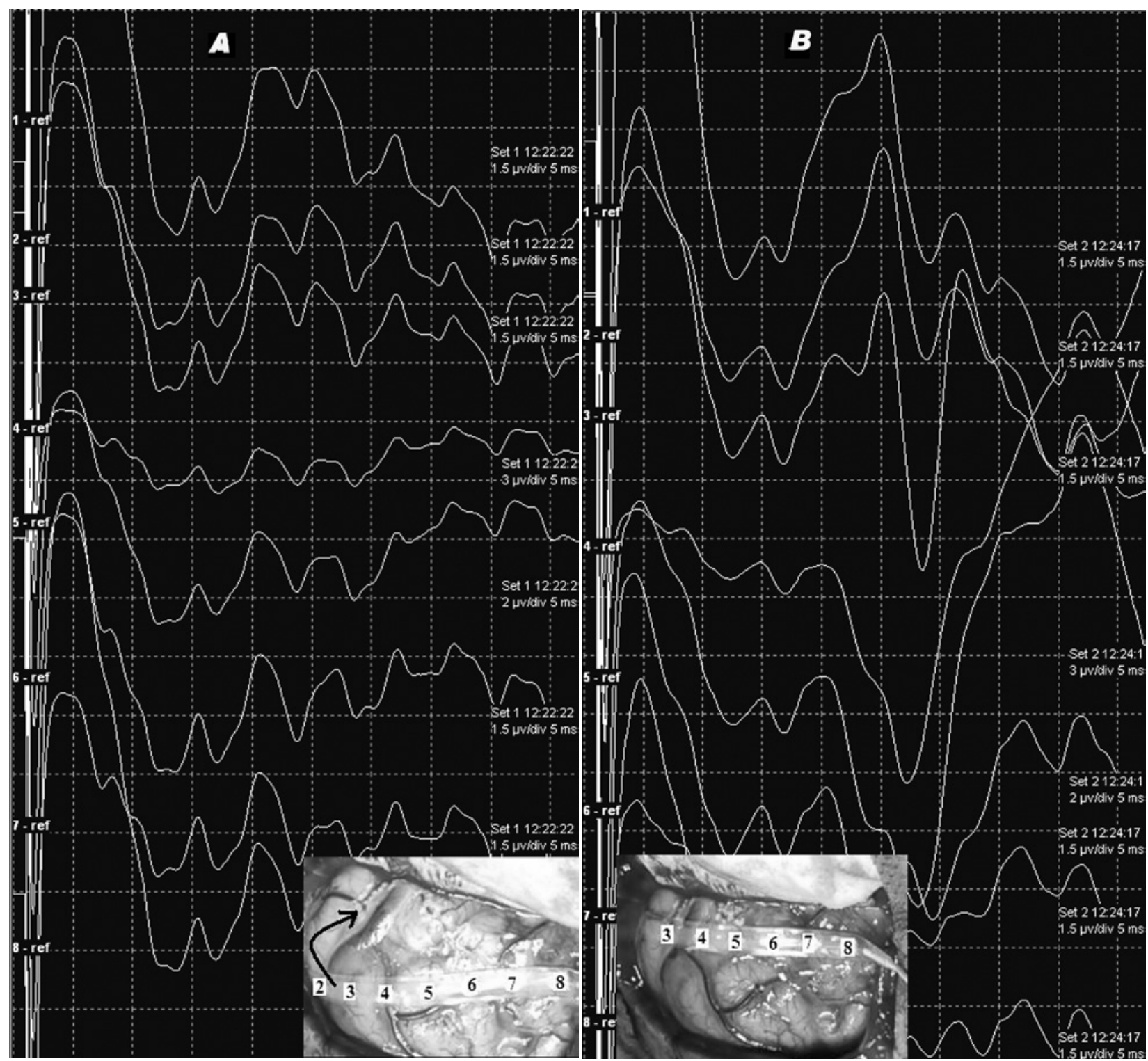

Figure 2: Repositioning of the recording subdural strip electrode with improvement of the morphology and amplitudes of the recorded cortical SSEPs as well as of their phase reversal. Panel A shows recording via a strip placed as shown in the picture. Contact 1 of the strip is the most posterior. No reliable SSEPs are recorded. Panel B shows SSEPs recordings after re-orentation of the strip, as shown by the arrow, in panel A. A clear phase reversal is seen between contacts 3 (postcentral) and 4 (precentral). Notice the new strip position. The sulcus seen between contacts 3 and 4 is identified as CS. 
Citation: Simon MV (2011) Intraoperative Neurophysiologic Sensorimotor Mapping-A Review. J Neurol Neurophysiol S3. doi:10.4172/2155-9562. S3-002

muscles. Alternatively, D waves recordings could also be done. Their main advantages there are robustness to anesthetics and high specificity; however, D wave recordings require an invasive procedure, as the recording electrode has to be inserted transcutaneously under fluoroscopic guidance in the spinal epidural space.

During the electrical cortical stimulation, continuous electrocorticogram (ECoG) recording is necessary in order to detect possible stimulation triggered afterdischarges (ADs) that can pose a safety risk to the patient as well as cause false positive mapping results.

\section{Technical details}

Stimulation: The surgeon performs cortical stimulation using a handheld stimulator, either monopolar or bipolar. We usually perform monopolar stimulation, with the monopolar handheld stimulator (Medronic Xomed, Inc-Prass standard monopolar stimulator probe) connected to the anode and a sterile needle (Viasys Healthcare, disposable subdermal needle, stainless steel, $12 \mathrm{~mm}$ length/27 G diameter) placed in the surgical field, as the cathode. This technique has evolved from the work of several authors $[26,26]$, who showed the advantage of cortical anodal stimulation. We use a multipulse train technique for stimulation [28]. This consists of applying repetitive trains of pulses, at 1-2 $\mathrm{Hz}$ frequency, each train consisting of 4-6 pulses at approximately $250 \mathrm{~Hz}$ (frequency of the pulses within the train) and with a $0.5 \mathrm{msec}$ pulse witdh. One of the advantages of this technique over the more extensively used modified Penfield technique first described by Berger et al. [3] (repetitive pulses of $0.5 / 1 \mathrm{msec}$ duration, at $50 / 60 \mathrm{~Hz}$ ) is that it allows direct electrophysiologic recording of the triggered mMEPs. Also, one should expect a lower mapping threshold and subsequently a more efficient and possibly safer motor mapping procedure [29]. Additionally, the stimulus artifact resulting from multipulse train stimulation is substantially less prominent and is more intermittent than in Penfield method, thus allowing an easier and earlier identification of possible ADs on ECoG.

We are starting the stimulation at $1 \mathrm{~mA}$, of all the regions of interest, before progressively increasing the intensity in $0.5 \mathrm{~mA}$ increments to a maximum of $25 \mathrm{~mA}$. Being able to predict the mapping threshold of the primary motor cortex, based on the patient's individual characteristics [23] will help increase the speed of this escalation and thus expedite the mapping procedure. The primary motor cortex is identified as the cortical region which stimulated at the lowest stimulus amplitude, triggers consistent, reproducible mMEPs in contralateral hemibody muscles, according to the Motor Homunculus (Figure 3). All cortical regions that stimulated resulted in mMEPs are further marked by the surgeon (Figure 1b).

Once the motor strip is identified, multipulse train stimulation can also be used during lesionectomy, for continuous functional monitoring

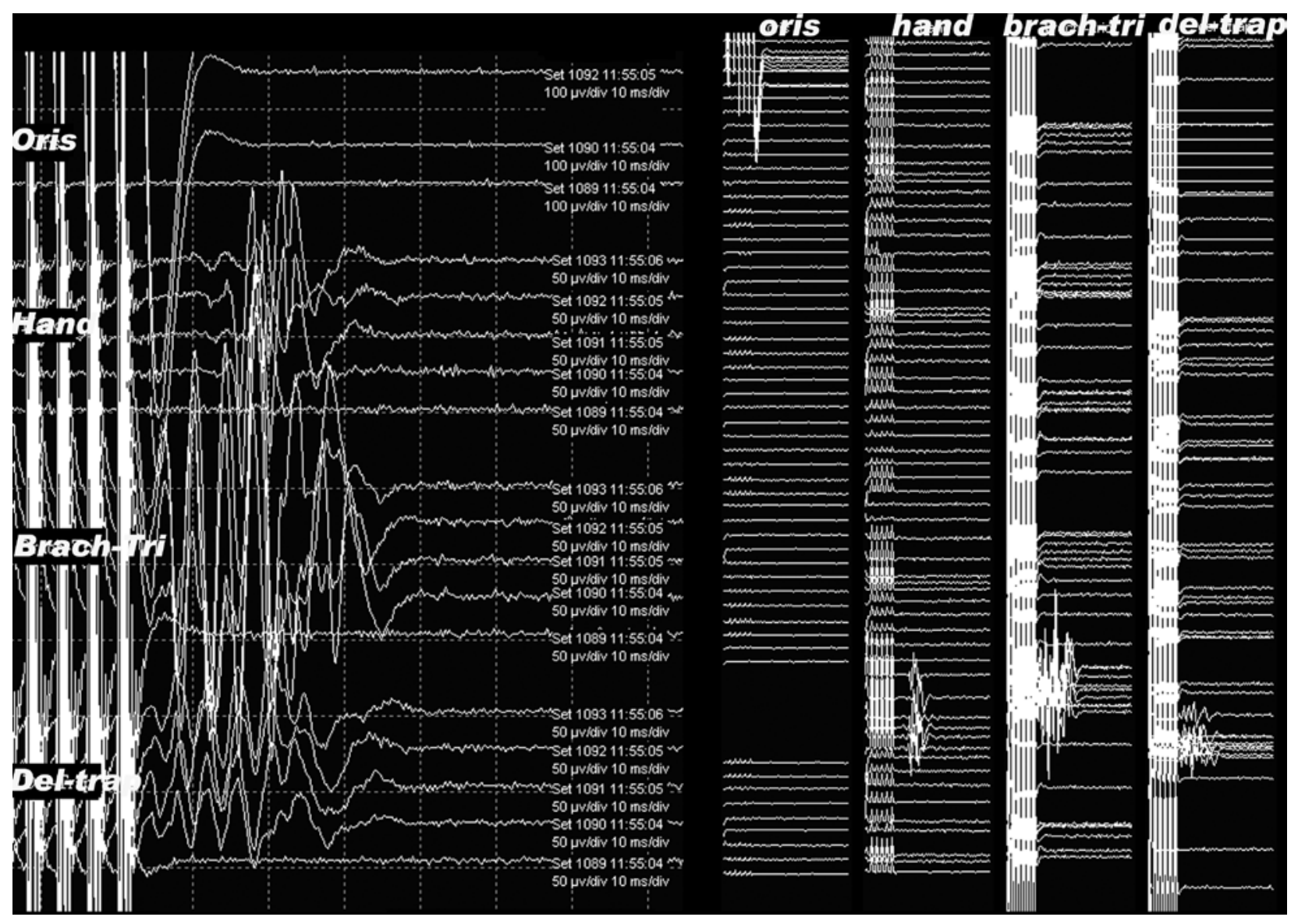

Figure 3: Triggered mMEPs by electrical stimulation of the Motor Homunculus. The left panel presents several muscle channels, each seen as a row of several trials each, with the most recent trials on top. The right panel shows a different display of the triggered mMEPs. There is one column for each muscle channel, with the most recent trials at the bottom. 
Citation: Simon MV (2011) Intraoperative Neurophysiologic Sensorimotor Mapping-A Review. J Neurol Neurophysiol S3. doi:10.4172/2155-9562. S3-002

of both the primary motor cortex as well as of the corticospinal tract, as described in section IV.

Recording the motor responses: As previously mentioned, we are using electrophysiologic recordings of the triggered contralateral (to the craniotomy side) hemibody motor responses (i.e., mMEPs). The recording electrodes are subdermal needless (XLTEK, disposable $13 \mathrm{~mm}$ length, $27 \mathrm{G}$ ) placed in the orbicularis oris, orbicularis oculi, masseter, trapezius, deltoid, triceps, brachioradialis, abductor policis brevis (APB), abductor digiti minimi (ADM), quadriceps (Quad), anterior tibialis (AT) and abductor halluces (AH). One channel can reflect the activity in two or in one muscle. Given the limitation introduced by the available number of recording channels, a "two muscles per channel" display, allows broader hemibody representation and thus a more sensitive recording. However, the other alternative (i.e., "one muscle per channel") results in increased specificity for appreciation of the location of the triggered mMEPs (i.e., which muscle) and indirectly of which part of the Motor Homunculus was stimulated. This becomes particularly useful in epidural cortical stimulator placement procedures. Additionally, recording from two adjacent electrodes, placed at a small distance in the same muscle and connected to one recording channel, will offer less noise, whereas electrodes situated at a longer distance (i.e., one electrode per muscle) will increase the risk of a noisy recording.

Alternatively, especially during awake craniotomies, subdermal needles can be replaced with surface electrodes (Rochester, ElectroMedical, disposable conductive solid gel electrodes). One could argue that, during awake procedures, neurophysiologic recordings are no longer necessarry as the patient can signal the occurrence of symptoms. However, triggered mMEPs occur at a lower stimulus threshold than symptoms or motor movements. Thus, we agree with other authors [30] that electrophysiologic recording should be done regardless of the patient's state (i.e., awake versus anesthetized).

Besides stimulation triggered mMEPs, limb movements related to stimulation triggered seizure activity can also be detected in the recording channels. This muscle activity is easily distinguished from triggered mMEPs and together with ECoG recordings (see section VI) can help to detect early seizure activity (Figure 4).

\section{Troubleshooting}

Inability to trigger mMEPs can have different causes. First, any possible technical reason should be ruled out. In general, lack of stimulus artifact signals faulty stimulation. Thus, the hardware and software set up for both stimulation and recording should be checked for errors. In case of stimulation using a handheld bipolar stimulator, one should make sure that its prongs do not touch. In case of using a monopolar stimulator, a needle electrode should be in place at the margin of the surgical field and it should be connected to the cathode. Last, defective handheld stimulators are not rare and thus replacing the stimulator should be the next step.

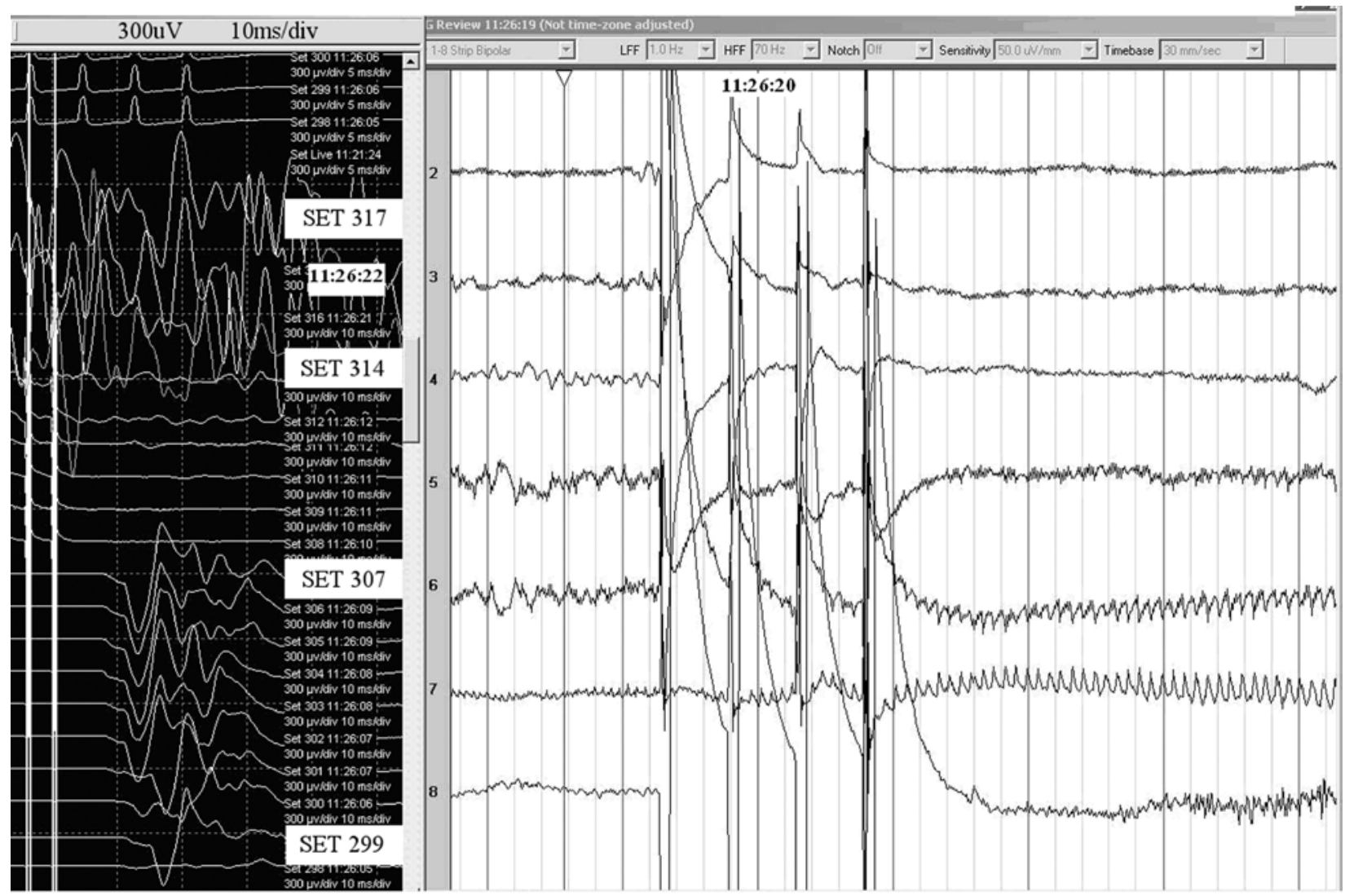

Figure 4: Electroclinical seizure onset, triggered by direct cortical stimulation. Clonic ictal movements of the contralateral hand translate in significant muscle artifact seen in the hand channel (sets 314 through 317). Earlier (sets 299 through 307), definite triggered mMEPs were obtained at the same stimulus intensity. The ECoG recording shows the electrical ictal onset at contacts 7 and 6 of the strip, consisting of rhythmic run of $12 \mathrm{~Hz}$ sharp activity. Muscle recording sensitivity is $300 \mathrm{uVI}$ div; timebase $10 \mathrm{msec} / \mathrm{div}$; ECoG recording has a sensitivity of $50 \mathrm{uV} / \mathrm{mm}$ and timebase is $30 \mathrm{~mm} / \mathrm{sec}$. 
Citation: Simon MV (2011) Intraoperative Neurophysiologic Sensorimotor Mapping-A Review. J Neurol Neurophysiol S3. doi:10.4172/2155-9562. S3-002

It is our experience that mMEPs in the face muscles are sometimes difficult to detect at the motor strip threshold. This is usually the case in the presence of significant amount of stimulus artifact in the recording channels, which will mask short latency, small amplitude facial mMEPs. An increase in stimulus intensity above the threshold may be necessary in order to trigger higher amplitude mMEPs; also one should attempt decreasing the stimulus artifact (e.g., replacing the ground electrode, changing the low frequency filter). A similar situation is encountered in case of noisy muscle channels, when the signal-to-noise ratio is reduced; again, an increase in the stimulus intensity will result in higher amplitude mMEPs that will help improving this ratio. We recommend that the recording electrodes impedances are less than $5 \mathrm{KOhms.}$

\section{Interpretation}

We consider as positive motor response mMEPs of at least $50 \mathrm{uV}$ peak to peak amplitude, and reproducible on subsequent stimulations of the same cortical regions. In general, polyphasic morphology signals a more robust activation; however, symptomatic patients may have simplified mMEPs morphologies at baseline, longer than expected absolute latencies and higher than expected stimulation thresholds.

For correct identification of the primary motor cortex, we strongly recommend finding its threshold and stimulating at this threshold. This is important for differentiating between primary and supplementary motor regions as it is known that damage of the motor strip will result in permanent postoperative motor deficit, while damage of the supplementary and premotor regions will cause temporary deficit (SMA syndrome). If high stimulus amplitudes are used, current spread to areas other than the one stimulated could trigger evoked responses "at distance" and subsequently falsely localize the entire primary motor cortex or parts of it (Figure 5).

Another challenge is differentiating stimulation triggered MEPs from spontaneous muscle activity that may have the expected latency for an evoked response. However, its random appearance on repeated stimulations of the same cortical region, or variability of this latency with repeated stimulation, should help in the differential diagnosis.

Last, stimulation triggered clinical motor seizure, may be reflected in muscle activity seen in the recording channels, and may occur at the same stimulation threshold as the triggered mMEPs. However, as previously specified, the morphology of these muscle activities differ; additionally, synchronization between ECoG and MEPs recordings helps in identifying their etiology (Figure 4).

\section{Continuous Monitoring of the Motor Pathways During Resection}

After identification of the motor strip, monitoring of the corticospinal tract and primary motor cortex during resection can be achieved by continuous stimulation of the latter and recording from the contralateral hemibody muscles (Figure 6). For this purpose, a second subdural strip electrode is placed directly on the motor strip and stimulation is done via two adjacent contacts (bipolar stimulation) or via one contact on the strip and the subdermal sterile needle placed at the margin of the surgical field (monopolar stimulation). A similar set up has been previously described [31]. The stimulus and recording parameters, as well as the recording set up for both mMEPs and ADs are identical with those used in the mapping. The stimulation is done at the motor strip threshold. A sudden decrease in the recorded mMEPs
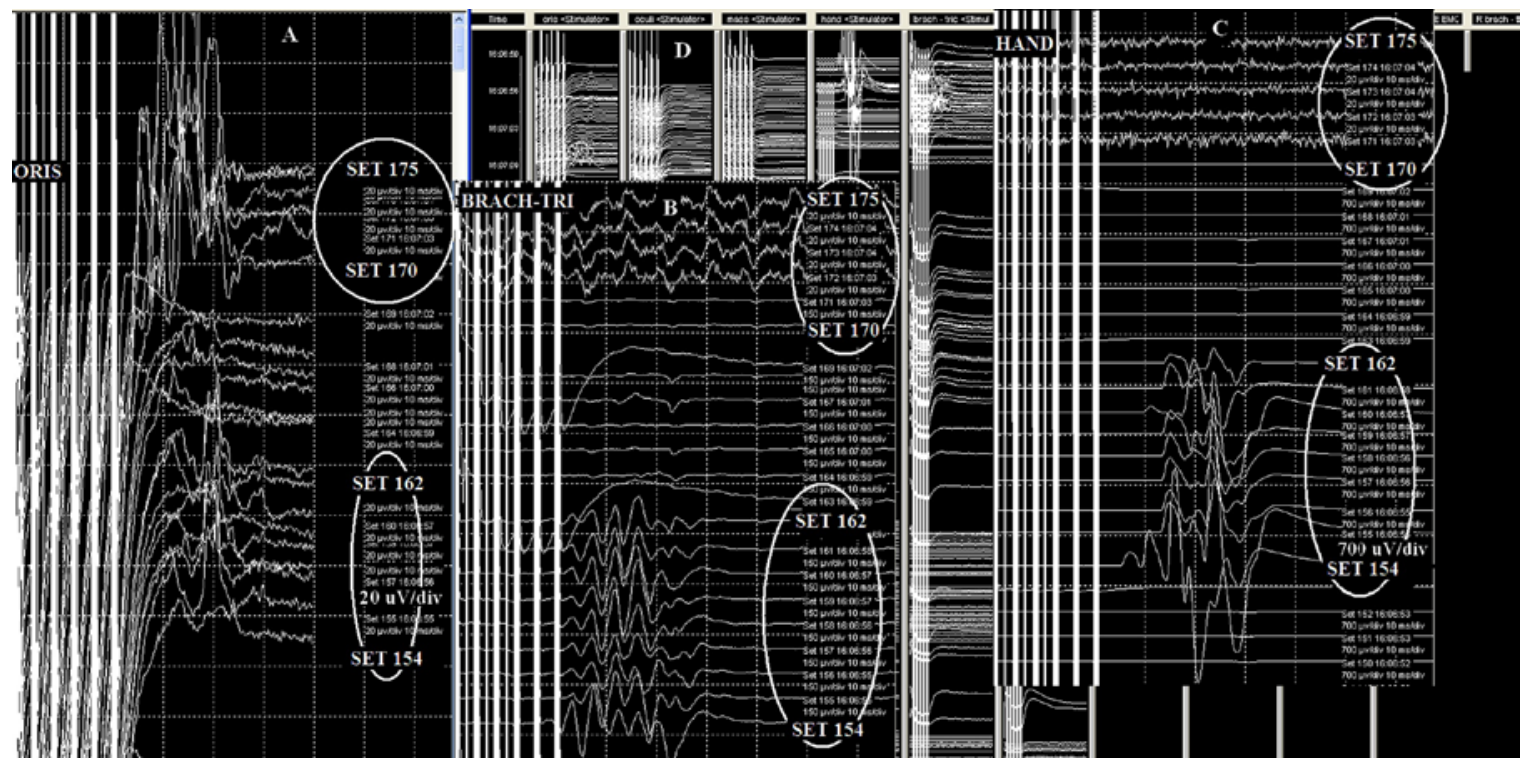

Figure 5: Stimulation above the primary motor cortex threshold causes current spread and loss of the specificity of stimulation. Panel A shows triggered mMEPs in the orbicularis oris channel triggered in two occasions: first time in sets 154 through 162 (bottom circle) and second time in sets 170 through 175 (top circle). However, panels B and C shows higher amplitude mMEPs also triggered in the brachioradialis-triceps and hand channels respectively, during sets 154-162. In these trials, the stimulation was done at current intensity (i.e. $7 \mathrm{~mA}$ ) higher than the mapping threshold. Thus, the activity seen in orbicularis oris channel in sets $154-162$ is most likely due to a spread of the current from the primary motor hand region. However, once the stimulus amplitude was decreased at 4 mA, stimulation of the face primary motor region, caused isolated mMEPs in the orbicularis oris channel (sets 170 through 175 in panel A) but not in the brachioradialis-triceps or hand channels ( see same sets in panels $\mathbf{B}$ and $\mathbf{C}$ respectively). Of note, the facial mMEPs in sets 170-175 have higher amplitude, more complex morphology and shorter latencies than the facial mMEPs triggered in sets 154 through 162, supporting the conclusion that in sets 170-175 direct (rather than indirect) stimulation of the face region occurred. Panel $\mathbf{D}$ in the background offers a chronological view of the overall mapping, with each column representing the activity in one muscle channel.

INTRAOPERATIVE CLINICAL NEUROPHYSIOLOGY by Mirela Simon. Copyright 2010 by DEMOS MEDICAL PUBLISHING LLC. Reproduced with permission of DEMOS MEDICAL PUBLISHING LLC in the format Other book via Copyright Clearance Center. 
Citation: Simon MV (2011) Intraoperative Neurophysiologic Sensorimotor Mapping-A Review. J Neurol Neurophysiol S3. doi:10.4172/2155-9562. S3-002

should bring the lesionectomy to immediate halt, irrigation with warm saline and to considering a different path of entry or of advancement of the resection. Using this method we were able to successfully guide maximal resection of precentral lesions, while avoiding postoperative neurologic deficit.

\section{Sensory Function Mapping and Monitoring}

Mapping of the primary somatosensory areas in the postcentral regions is usually done with the patient awake so that he or she can communicate presence or absence of sensory symptoms triggered by electrical stimulation.

However, both in awake and anesthetized patients, somatosensory cortex can be reasonably well identified by recording SSEPs triggered by stimulation of different peripheral nerves [32]. The method has been already described in section II. Subsequently, continuous stimulation of these nerves with continuous recording of the resulting SSEPs, will allow monitoring of the integrity of the somatosensory cortex during lesionectomy. A sudden change in the amplitude, morphology and/or latency of the recorded SSEPs should raise the question of dysfunction of the thalamocortical sensory pathways.

\section{Monitoring of ADs}

As previously mentioned, during the cortical electrical stimulation and using ECoG recordings allow continuous monitoring of ADs (Figure 4). For the purpose, the 8 contact subdural electrode strip that had been used for SSEPs phase reversal technique is now repositioned in close proximity to the cortical regions to be stimulated, and the inputs are connected to the amplifier head box of an EEG machine. Continuous recording is done via both bipolar and referential montages (reference electrode placed on the contralateral mastoid). The recording parameters are the same as for a regular EEG recording.

Of note, EcoG recording enables not only identification of ADs but also appreciation of the depth of anesthesia by analyzing the depth of the EEG burst suppression pattern. If the bursts of electrical brain activity are separated by long periods of isoelectric EEG, higher motor thresholds are to be expected [22,23]. Thus, the escalation in the stimulus amplitude can be done faster.

Additionally, the recording allows us to appreciate the degree of abnormal cortical excitability present at baseline, particularly in epilepsy as well as in brain tumor patients who have experienced clinical seizures preoperatively. This is an important information, as further electrical stimulation of an already hyperexcitable cortex could trigger ADs and seizures more easily. Thus, additional intravenous boluses of antiepileptic drugs (AEDs) such as Phosphentoin and Leviteracetam (Keppra) may be considered.

Cortical irrigation with ice cold saline is an efficient method to abort the stimulation triggered ADs [33] and so, before the stimulation is initiated, one should make sure that ice cold saline is available for this purpose.
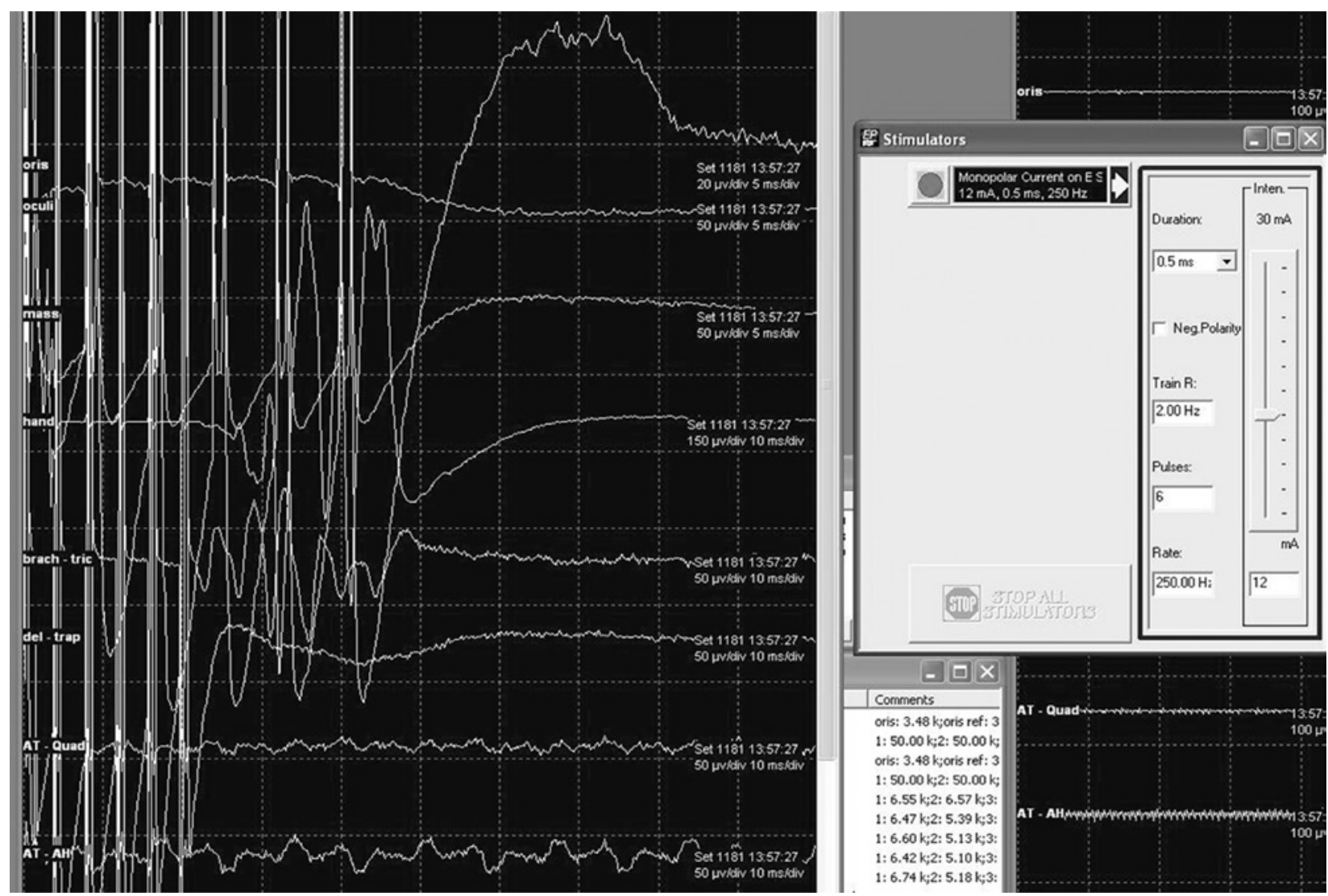

Figure 6: Continuous mMEPs monitoring during lesionectomy in the precentral gyrus. The stimulation is done via one contact of a subdural strip (anode) and a sterile needle electrode placed at the margin of the surgical field (cathode). Noticed polyphasic mMEPs triggered in the hand and brachioradialis-triceps channels. The stimulation is done with repetitive trains at $2 \mathrm{~Hz} ; 6$ pulses/trains, pulse width $0.5 \mathrm{msec}$ and pulses' frequency within each train at $250 \mathrm{~Hz}$. 
Citation: Simon MV (2011) Intraoperative Neurophysiologic Sensorimotor Mapping-A Review. J Neurol Neurophysiol S3. doi:10.4172/2155-9562. S3-002

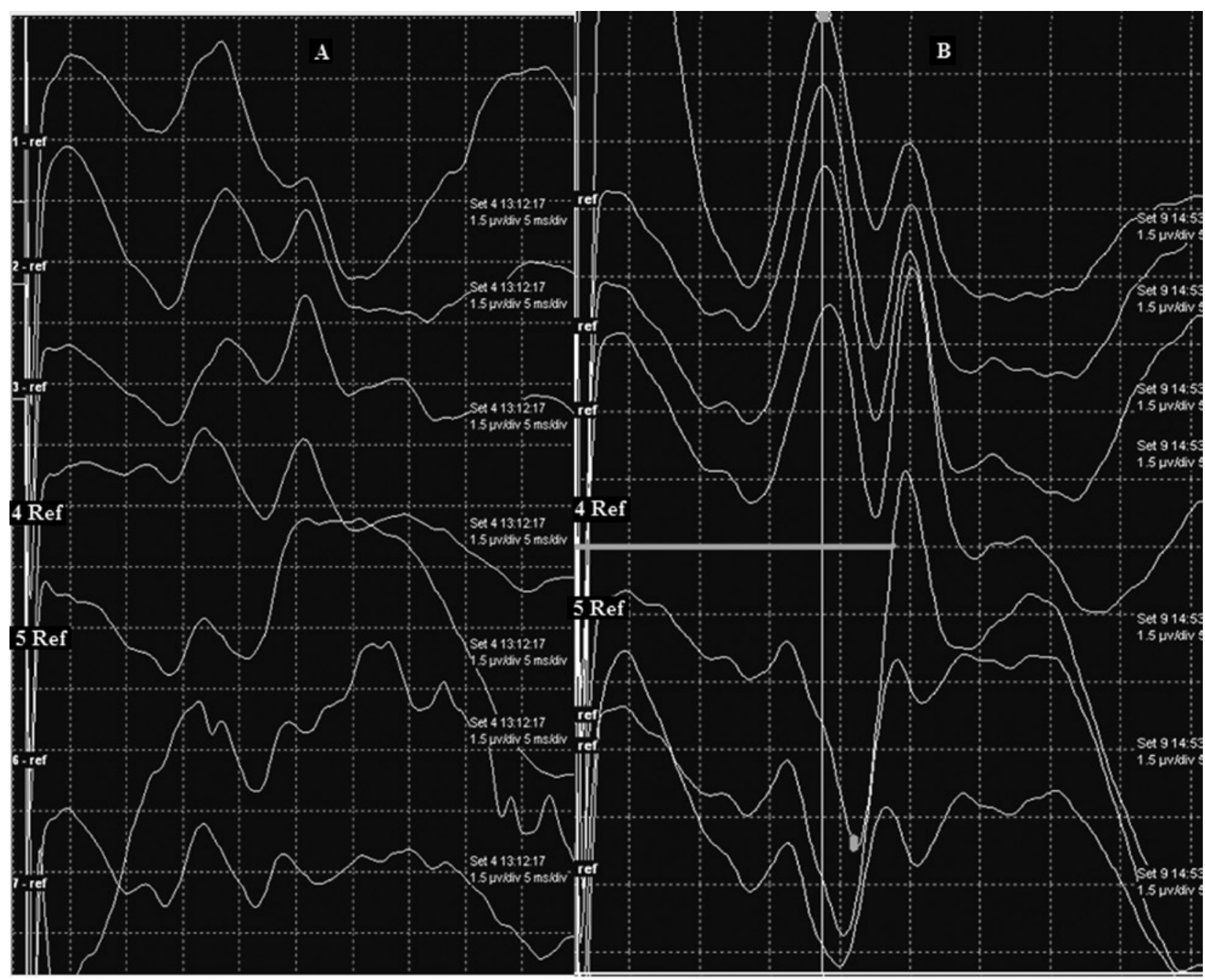

Figure 7: Median SSEPs phase reversal technique: anesthetic induced changes. Panel A shows cortical SSEPs recorded via an 8 contact strip, during anesthesia with nitrous oxide and sevoflurane: the exact location of N19/P22 potentials as well as phase reversal of the recorded dipole are hard to appreciate. Panel B shows recording via the same strip, in the same position, after the anesthetic regimen has been changed to TIVA. Notice a clear phase reversal of recorded SSEPs (line) at the level of the central sulcus, placing contact 4 in the postcentral region and contact 5 in the precentral region.

\section{Anesthetic Requirements}

It is well known that inhalational agents such as nitrous oxide and halogenated agents do have a significant negative impact on generation of SSEPs, especially when used together [22,34]. Thus, during SSEPs phase reversal technique their use should be limited as much as possible. While low concentrations of inhalational agents may be acceptable in asymptomatic patients, they should be avoided altogether in patients who are experiencing sensory symptoms, as in these patients one expects poor baseline SSEPs. The use of inhalational agents may significantly distort the morphology of the thalamocortical responses and hinder detection of a phase reversal (Figure 7). However, their impact may be even more significant, to total annihilation of identifiable waveforms.

Similarly, triggered mMEPs by direct cortical stimulation are significantly affected by nitrous oxide and halogenated agents, due to different mechanisms, from decreased cortical excitability and delayed axonal transmission in the corticospinal tracts, to decreased synaptic transmission at the level of the spinal alpha motor neuron [35-41].
In summary, during SSEPs phase reversal technique and direct electrical stimulation for sensorimotor mapping, the use of inhalational agents should be limited as much as possible and instead total intravenous anesthesia (TIVA with, for example, propofol and remifentanyl) should be used. Last, during TIVA one should avoid a deep level of anesthesia, translating into a deep EEG burst suppression pattern [42] and which may result in an unreasonably high motor threshold [22,23,34].

\section{References}

1. Albright AL, Wisoff JH, Zeltzer P, Boyett J, Rorke LB, et al. (1995) Prognostic factors in children with supratentorial (nonpineal) primitive neuroectodermal tumors. A neurosurgical perspective from the Children's Cancer Group. Pediatr Neurosurg 22: 1-7

2. Ammirati M, Vick N, Liao YL, Ciric I, Mikhael M (1987) Effect of the extent of surgical resection on survival and quality of life in patients with supratentorial glioblastomas and anaplastic astrocytomas. Neurosurgery 21: 201-206.

3. Berger MS, Kincaid J, Ojemann GA, Lettich E (1989) Brain mapping techniques to maximize resection, safety, and seizure control in children with brain tumors. Neurosurgery 25: 786-792.

4. Berger MS (1996) The impact of technical adjuncts in the management of 
Citation: Simon MV (2011) Intraoperative Neurophysiologic Sensorimotor Mapping-A Review. J Neurol Neurophysiol S3. doi:10.4172/2155-9562. S3-002

cerebral hemispheric low- grade gliomas of childhood. J Neurooncol 28: 129155.

5. Guilburd JN, Lapras C, Guyotat J (1989) Brain tumors in infants. Harefuah 116: 133-135.

6. Nitta T, Sato K (1995) Prognostic implications of the extent of surgical resection in patients with intracranial malignant gliomas. Cancer 75: 2727-2731.

7. Sala F, Krzan MJ, Deletis V (2002) Intraoperative neurophysiological monitoring in pediatric neurosurgery: why, when, how?. Child's Nervous System 18: $264-$ 287.

8. Scerrati M, Roselli R, lacoangeli M, Pompucci A, Rossi GF (1996) Prognostic factors in low-grade gliomas of the cerebral hemispheres: the role of surgery. $J$ Neurol Neurosurg Psychiatry 61: 291-296.

9. Yang HJ, Nam DH, Wang KC, Yeon Mee Kim, Chi JG et al. (1999) Supratentoria primitive neuroectodermal tumor in children: clinical features, treatment outcome and prognostic factors. Childs Nerv Syst 15: 377-383.

10. Bonilha L, Kobayashi E, Mattos JP, Honorato DC, Li LM, et al. (2004) Value of extent of hippocampal resection in the surgical treatment of temporal lobe epilepsy. Arq Neuropsiquiatr 62: 15-20.

11. Kim SK, Wang KC, Hwang YS, Kim KJ, Chae JH, et al. (2008) Epilepsy surgery in children: outcomes and complications. J Neurosurg Pediatr 1: 277-283.

12. Paolicchi JM, Jayakar P, Dean P, Yaylali I, Morrison G, et al. (2000) Predictors of outcome in pediatric epilepsy surgery. Neurology 54: 642-647.

13. Ojemann JG, Miller JW, Silbergeld DL (1996) Preserved function in brain invaded by tumor. Neurosurgery 39: 253-259.

14. Schiffbauer H, Ferrari P, Rowley HA, Berger MS, Roberts TP (2001) Functiona activity within brain tumors: a magnetic source imaging study. Neurosurgery 49: $1313-1320$

15. Skirboll SS, Ojemann GA, Berger MS, Lettich E, Winn HR (1996) Functiona cortex and subcortical white matter located within gliomas. Neursurgery 38 678-685.

16. Korvenoja A, Kirveskari E, Aronen HJ, Avikainen S, Brander A, et al. (2006) Sensorimotor cortex localization: comparison of magneto encephalography, functional MR imaging and intraoperative cortical mapping. Radiology 241 213-222.

17. Meyer FB, Bates LM, Goerss SJ, Friedman JA, Windschitl WL, et al. (2001) Awake craniotomy for aggressive resection of primary gliomas located in eloquent brain. Mayo Clin Proc 76: 677-687.

18. Kombos T, Süss O, Vajkoczy P (2009) Subcortical mapping and monitoring during insular tumor surgery. Neurosurg Focus 27: E5.

19. Duffau H, Capelle L, Sichez N, Denvil D, Lopes M, et al. (2000) Intraoperative mapping of the subcortical language pathways using direct stimulation. Brain 125: 199-214.

20. Lehéricy S, Duffau H, Cornu P, Capelle L, Pidoux B, et al. (2000) Correspondence between functional magnetic resonance imaging somatotopy and individual brain anatomy of the central region: comparison with intraoperative stimulation in patients with brain tumors. J Neurosurg 92: 589-598.

21. Bitar RG, Olivier A, Sadikot AF, Andermann F, Reutens DC (2000) Cortica motor and somatosensory representation: effect of cerebral lesions. J Neurosurg 92: 242-248.

22. Simon MV, Shields DC, Eskandar EN Functional Cortical Mapping. In: Simon MV, ed.Intraoperative neurophysiology: A Comprehensive guide to monitoring and mapping. Demos Medical Publishing, New York, USA.

23. Simon MV, Michaelides C, Wang S, Chiappa KH, Eskandar EN (2010) The effects of EEG suppression and anesthetics on stimulus thresholds in functional cortical motor mapping. Clin Neurophysiol 121: 784-792.

24. Kombos T, Suess O, Funk T, Kern BC, Brock M (2000) Intraoperative mapping of the motor cortex during surgery in and around the motor cortex. Acta Neurochir 142: 263-268.

25. Patton HD, Amassian VE (1954) Single and multiple-unit analysis of cortical stage of pyramidal tract activation. J Neurophysiol 17: 345-363.

26. Hern JE, Landgren S, Phillips CG, Porter R (1962) Selective excitation of corticofugal neurones by surface-anodal stimulation of the baboon's moto cortex. J Physiol 161: 73-90.
27. Ranck JB Jr (1975) Which elements are excited in electrical stimulation in mammalian central nervous system: a review. Brain Res 98: 417-440.

28. Neuloh G, Schramm J (2002) Mapping and monitoring of supratentorial procedures. In: Deletis and Shills, eds: Neurophysiology in Neurosurgery: Modern Intraoperative Approach. Academic Press, Sand Diego, USA

29. Taniguchi, Makoto, Cedzich, Cornelia, Taniguchi, et al. (1993) Modification of cortical stimulation for motor evoked potentials under general anesthesia: technical description. Neurosurgery 32: 219-226.

30. Yingling CD, Ojemann S, Dodson B, Harrington MJ, Berger MS, et al. (1999) Identification of motor pathways during tumor surgery facilitated by multichanne electromyographic recording. J Neurosurg 91: 922-927.

31. Suess O, Suess S, Brock M, Kombos T (2006) Intraoperative electrocortica stimulation of Brodman area 4: a 10-year analysis of 255 cases. Head Face Med 2: 20

32. Kumabe T, Nakasato N, Nagamatsu K, Tominaga T (2005) Intraoperative localisation of the lip sensory area by somatosensory evoked potentials. J Clin Neurosci 12: 66-70.

33. Sartorius CJ, Berger MS (1998) Rapid termination of intraoperative stimulationevoked seizures with application of cold Ringer's lactate to the cortex. Technical note. J Neurosurg 88: 349-351.

34. Simon MV (2010) The Effects of Anesthetics on Intraoperative Neurophysiology Studies. In: Simon MV, ed. Intraoperative neurophysiology: A Comprehensive guide to monitoring and mapping. Demos Medical Publishing, New York, USA

35. Burke D, Bartley K, Woodforth IJ, Yakoubi A, Stephen JP (2000) The effects of volatile anesthetics on the excitability of human corticospinal axons. Brain 123: $992-1000$

36. Haghighi SS (1998) Influence of isoflurane anesthesia on motor evoked potentials elicited by transcortical, brainstem and spinal root stimulation. Neurol Res 20: 555-558.

37. Hentschke H, Schwarz C, Antkowiak B (2005) Neocortex is the major targe of sedative concentrations of volatile anesthetics: strong depression of firing rates and increase of GABAA receptor-mediated inhibition. Eur J Neurosci 21 93-102.

38. Hicks R, Burke D, Stephen J, Woodforth I, Crawford M (1992) Corticospinal volleys evoked by electrical stimulation of human motor cortex after withdrawa of volatile anaesthetics. J Physiol 456: 393-404.

39. Loughnan BA, Anderson SK, Hetreed MA, Weston PF, Boyd SG, et al. (1989) Effects of halothane on motor evoked potentials recorded in the extradural space. Br J Anaesth 63: 561-564.

40. Osawa M, Shingu K, Murakawa M, Adachi T, Kurata J, et al. (1994) Effects of sevoflurane on central nervous system electrical activity in cats. Anesth Analg 79: 52-57.

41. Zentner J, Albrecht T, Heuser D (1992) Influence of halothane, enflurane, and isoflurane on motor evoked potentials. Neurosurgery 32: 298-305.

42. Kortelainen J, Koskinen M, Mustola S, Seppänen T (2007) EEG frequency progression during induction of anesthesia: from start of infusion to onset of burst suppression pattern. Conf Proc IEEE Eng Med Biol Soc 1: 1570-1573.

43. Fandino J, Kollias SS, Wieser HG, Valavanis A, Yonekawa Y (1999) Intraoperative validation of functional magnetic resonance imaging and cortical reorganization patterns in patients with brain tumors involving the primary motor cortex. J Neurosurg 91: 238-250.

44. Lesser RP, Arroyo S, Crone N, Gordon B (1998) Motor and sensory mapping of the frontal and occipital lobes. Epilepsia 39: S69-S80.

45. Mavroudakis N, Vandesteene A, Brunko E, Defevrimont M, Zegers de Beyl D (1994) Spinal and brainstem SEPs and $\mathrm{H}$ reflex during enflurane anesthesia. Electroencephalogr Clin Neurophysiol 92: 82-85.

46. Shaul N (1998) The fundamental neural mechanisms of electroencephalography Electroencephalography Clinical Neurophysiology 106: 101-107.

47. Stecker MM, Kramer TH, Raps EC, O'Meeghan R, Dulaney E, et al (1998) Treatment of refractory status epilepticus with propofol: clinical and pharmacokinetic findings. Epilepsia 90: 18-26.

48. Tan TC, Black PM (2001) Awake craniotomy for excision of brain metastase involving eloquent cortex techniques in neurosurgery. 7: 85-90. 